\title{
Alternative Optical Acquisition Technique for Supporting Reverse Engineering Process
}

\author{
Suchada Rianmora and Molticha Rangsiyangkoon
}

\begin{abstract}
In product development phase, Reverse Engineering (RE) can help to rapidly create the design of the new product while satisfying the customer's satisfaction. Optical acquisition technology can be applied in data acquisition phase of $\mathrm{RE}$ process to minimize time and number of activities. Time spent for accomplishing RE process and the accuracy of the surface finish (3D virtual model) depend upon the method selected for acquiring an entire surface of an existing part. This research introduces the acquisition system by using optical acquisition technologies where 3D laser scanner, displacement sensor, and digital camera have been presented and discussed.
\end{abstract}

Index Terms-Laser processing technology, reverse engineering (re), data acquisition, optical signal processing, and 3d triangulation process.

\section{INTRODUCTION}

Laser Processing is introduced as one of the optical signal processing technologies that can develop the performance of design phase in manufacturing process. Laser applications advance to manufacturing tools such as laser welding, laser cutting, laser optics design, laser heat treatment, and laser scanning [1]. The signal processing makes a deal with transferring information methods in many different formats, e.g., physical, symbolic, and abstract, wildly known as signals [2]. Reverse Engineering (RE) applies the concept of data acquisition system for constructing 3D CAD model. To support RE process, recently, the 3D laser scanning technology has been applied to acquire material surface. This method can rapidly collect the geometry shape from physical object. Many researchers have studied on advantages and disadvantages of laser scanning techniques. Although, the laser scanner provides high precision of 3D mesh design, it contains some noises on the 3D virtual model. This results as the unorganized output which need to be eliminated. This technique is very sensitive to the light intensity and light reflection [3], object size, and types of the object surfaces (e.g., transparent, fluorescent, shiny, glossy or black color) the extra activities as coating surface of the object or attaching the references on the master model are required. These disadvantages have the direct effects on time consuming process and high cost of operation. There are three optical acquisition techniques that have been introduced in this research which are commercial 3D laser scanner, in-house Automated Selective Acquisition System (ASAS) [4], and the proposed technique. In ASAS technique, the obtained results are presented as a series of contour points

Manuscript received March 3, 2016; revised July 19, 2016.

The authors are with Thammasat University, Thailand (e-mail: suchada@siit.tu.ac.th, molticha.lee@gmail.com). which represent the geometric shapes of the entire object. It is fast acquisition process; however, the sizes and some shapes of the object are limited. It would be better to develop a compact device which can acquire and support various sizes and geometric shapes. This paper presents an alternative image-based processing technique to quickly capture the details of a master object with less human labour required where a digital camera is the key component of this developed technique.

\section{RELATED WORKS}

This section presents 3 optical technologies used for data acquisition phase in RE process.

\section{A. Image Processing}

Image processing is a technique used for converting an image to be digital form [5]. Normally, image processing includes three steps as importing the image, analyzing the image, and reporting the analysis. The aim of image processing can be classified 5 groups as visualization [6], image sharpening and restoration [7], image retrieval [8], measurement of pattern [9], and image recognition [10].

\section{B. Reverse Engineering}

Reverse Engineering is process that duplicates existing objects to see how it works [11]. Nowadays, reverse engineering software package was applied into industrial process, such as using 3D scanner in computer aided inspection [12], analyzing FEM in aerospace turbine blade [13], and managing file system in CCTC [14].

\section{3D Technology}

3D information has become an important factor in wildly fields of works, i.e., medical simulation [15], [16], animation [17], [18], graphic design [19], animation [20], entertainment [21], and agriculture [22]. 3D technology was found in 1838 by Sir Charles Wheatstone who described the process of stereopsis [23]. Based on the computer graphics, 3D modeling is the process of displaying 3D surface of product [24]. The differentiation of 3D modelling that is better than 2D method is flexibility of showing realistic shape which provides more accuracy result and reduces human error in misplacing.

\section{RESEARCH CONCEPT}

Concept of this research is to reconstruct 3D model from a simple product by using three different acquisition processing techniques (figure1) which are Commercial Laser Scanner (laser processing), Automated Selective Acquisition System or ASAS (displacement sensor processing) [5], and 
Digital Camera (image-based acquisition). The proposed approach aims to identify the appropriate acquisition technique for extracting geometric shapes of an object. Some objects that contain glossy surface, or small size are quite difficult to be captured by laser sources, whereas, some object can be easily to be detected. The obtained results from each optical technique will be compared and discussed. The sample product used in this research is white wine glass with 52-mm.-height size.

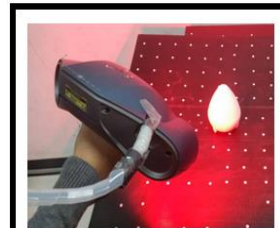

(a)

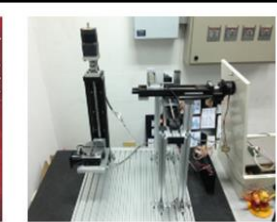

(b)

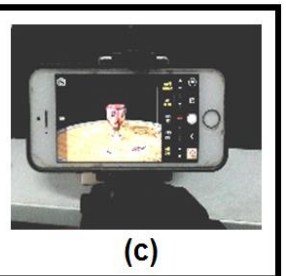

(c)
Fig. 1. Three acquisition devices: (a) 3D laser scanner, (b) ASAS, and (c) digital camera.

\section{RESEARCH METHODOLOGY}

According to accomplish the aim of this proposed research which is about comparing the three different acquisition techniques to quickly reconstruct 3D model, four main steps are required.

\section{A. Preparing Sample Surface}

Before extracting geometric shapes of a sample object that contains glossy, transparent or fluorescence surface, the surface preparation is required for reducing errors from data acquisition process (Fig. 2). In the process, wine glass was coated by powder spray with flat white color for removing glossy surface. For applying digital camera, types of object's shape (i.e., symmetrical or non-symmetrical shape) and surface color (i.e., multiple colors or single color) are the main issues that have the direct effects to the accuracy of the obtained virtual model. For an object that contains symmetrical shape with single color, the additional multi-color printings and stickers are attached on the object's surface.

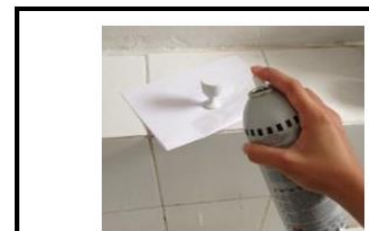

(a)

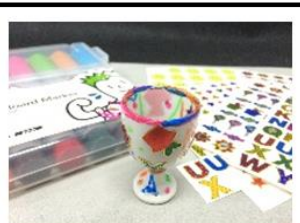

(b)
Fig. 2. Surface preparation process: (a) coating, and (b) adding sticker and painting.

\section{B. Preparing Background/Environment}

After preparing the object surface, for 3D laser scanner, the white-circular reference points which are located as the chess pattern (Fig. 3(a)) are added on the black board. For ASAS technique, setting the position of the object and the laser spot is required where the laser spot is set at the center of the object (Fig. 3(b)) before scanning attempt. For digital camera, in order to obtain clean and clear virtual model, the rotary platform used for locating an object is designed to be multi-color patterns with various graphic artworks (Fig. 3(c)). These additional references can help the camera quickly detecting shapes of the object and reduce time spent for combining images to form an accurate virtual shape in the subsequent process, the image registration process, since the common positions are shown on each frame taken. The distorted regions and the missed features of the merged model can be eliminated.

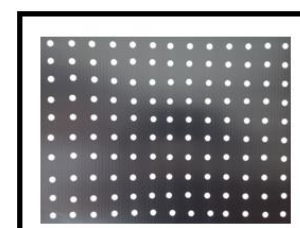

(a)

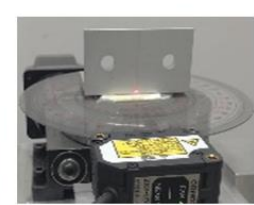

(b)

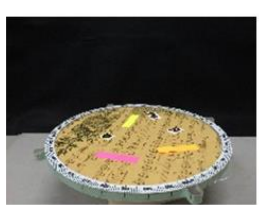

(c)
Fig. 3. Preparing background: (a) White-circular reference points on black background, (b) Projected red laser spot on the object's center, (c) Multi-colors printing papers/sticker on the rotary platform.

\section{Acquiring Process}

In 3D laser scanner application, it provides the unorganized point cloud data representing product's shape (Fig. 4(a)). For obtaining the organized data points, automated selective acquisition system (ASAS) is applied where the object's surface is acquired layer by layer from the bottommost to the topmost area. The data reduction process is not required. The detected distance between the laser source and the object's surface is called a point (Fig. 4(b)). For the proposed technique, the concept of image-based acquisition is applied where a camera is used to capture the entire object's surface in different views. The results of capturing activity present as a series of images which are then registered into a 3D mesh model (Fig. 4(c)).

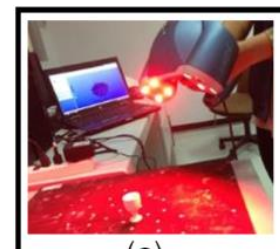

(a)

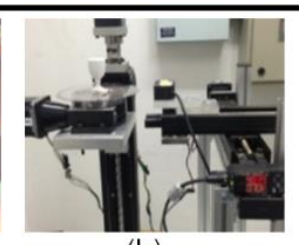

(b)

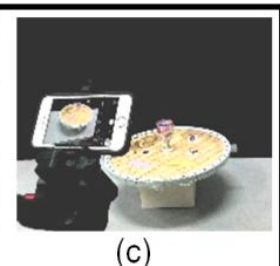

(c)
Fig. 4. Acquiring Process: (a) 3D laser scanner, (b) ASAS, and (c) digital camera.

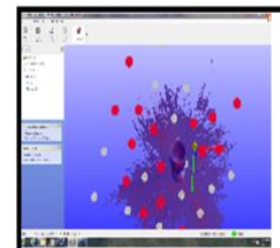

(a)

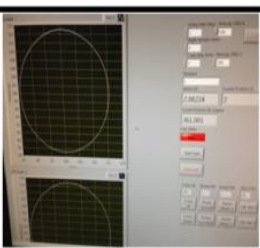

(b)

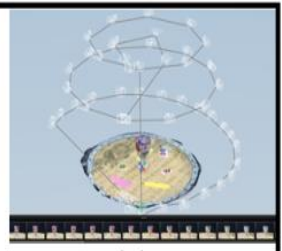

(c)
Fig. 5. Reconstructing 3D model: (a) 3D laser scanner, (b) ASAS, and (c) digital camera.

\section{Reconstruct 3D Model}

After The laser scanner requires data reduction process before reconstructing surfaces since some noises (i.e., unwanted regions) are shown. Then the complete triangular facets are formed as 3D CAD model (Fig. 5(a)). In ASAS process, the organized and contour points can be used to construct 3D CAD model directly and quickly. However, the bottommost and the topmost areas cannot be detected because of the limitation of ASAS machine (Fig. 5(b)), and, sometimes, the multiple reflections of a laser source are occurred. For the last one, a series of images are combined 
where the common points (i.e., the same reference stickers or printing papers shown on each frame taken) are registered to form a virtual model (Fig. 5(c)).

\section{RESULT AND ANALYSIS}

Three obtained models (Fig. 6) from three acquisition techniques were compared where the result obtained from the proposed approach was used as the reference. In 3D laser scanner, the scanning head was moved freely and randomly to acquire entire object's surface. Once the regions were completely acquired, the scanning process had been stopped for a while for storing the data. This consumed 10 to 15 seconds for simulating and representing the obtained data in the point forms. The scanned surfaces contained some noises which were eliminated by the redundant activity, data reduction process. Time required for accomplishing the surface reconstruction task of this technique was higher than the proposed technique's.

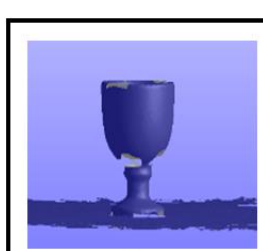

(a)

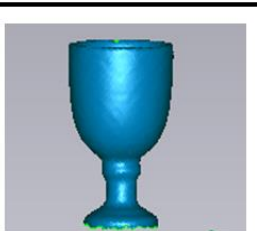

(b)

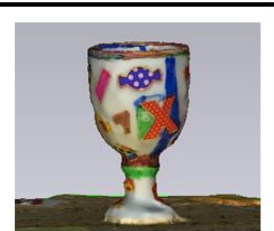

(c)
Fig. 6. Obtained 3D model: (a) 3D laser scanner, (b) ASAS, and (c) digital camera.

For ASAS technique, when the scanning parameters which were height, speed of all $\mathrm{X}, \mathrm{Y}, \mathrm{Z}$ and c-axes, speed of distance sensor, and the angle between 2 points, were completely assigned to the system, the rotary table started to turn while the laser spot was detecting the object's surface from the bottommost contour. When the current scanning contour was done (a complete 360-degree turn), the next scanning process was started where the platform (Z-axis) was moved down for $1 \mathrm{~mm}$ (i.e., scanning resolution was $1 \mathrm{~mm}-$ the distance between the two consecutive contours). The acquiring activity kept performing until the topmost level of the object was reached, and time spent for each scanning revolution was 20 seconds. Since the length of wine glass was $52 \mathrm{~mm}$, thus the total time spent for accomplishing this step was about 18 minutes.

In digital camera technique, it required about 70 images taken from the object at 70 different views to completely reconstruct the entire geometric shape of the virtual model. Each snap shot took 3 seconds. Thus this process totally spent 3.5 minutes. Comparing to the ASAS technique, the proposed system provided shorter scanning time to obtain the entire surface details where less human was required.

The noises (i.e., unwanted regions of the acquired data) can be eliminated by using the commercial surface reconstruction application (Fig. 7), and the final 3D mesh models of three acquisition techniques are shown in Fig. 8. The process of creating mesh or triangular facets was started.

The error can be identified as the distances of nearest points between the obtained model and the master part (i.e., reference model) are computed. In order to compute the distance for each point of the compared cloud, the method called the 'nearest neighbor distance' is applied. The results of cloud compared are shown in Fig. 9. In this research, according to Eq.1, if the distance of any compared points is greater than $0.05 \mathrm{~mm}$ (assigned by the error size that should not exceed $1 \%$ of wine glass height), these will be considered as outliers. Illustrated in Table I are the point comparisons after eliminating noises where the percentage of error $(\% \mathrm{E})$ of each acquisition technique was calculated by comparing the number of outlier points with the number of total points of master model which are 890,478 points, when the length of this wine glass is $52.5 \mathrm{~mm}$.

TABLE I: THE FINAL RESUlTS AFTER ELIMINATING NOISES

\begin{tabular}{lcc}
\hline Techniques & $\begin{array}{c}\text { Outlier } \\
\text { Points }\end{array}$ & $\begin{array}{c}\text { Errors }(\%) \\
\text { (Compared with master model) }\end{array}$ \\
$\begin{array}{l}\text { 1. Laser } \\
\text { scanning }\end{array}$ & 136,000 & $(136,000 / 890,478) \times 100=15.27$ \\
2. ASAS & 75,000 & $(75,000 / 890,478) \times 100=8.42$ \\
3. Camera & 145,000 & $(145,000 / 890,478) \times 100=16.28$ \\
\hline
\end{tabular}

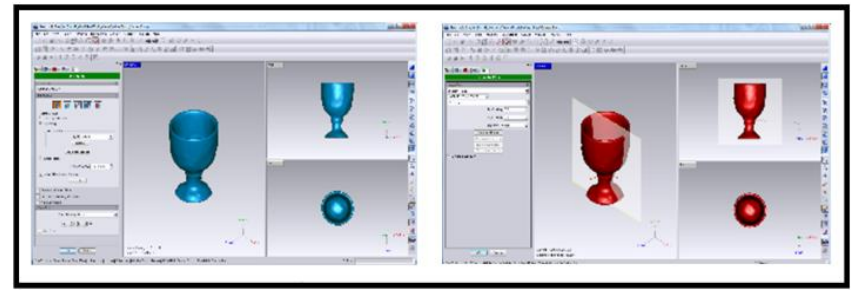

Fig. 7. The eliminating noises from the obtained model by using surface reconstruction software.

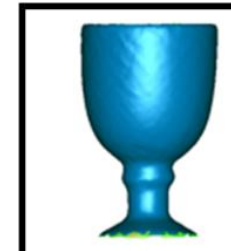

(a)

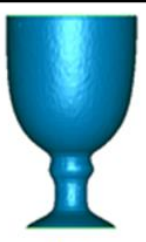

(b)

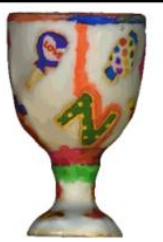

(c)
Fig. 8. The final 3D model: (a) 3D laser scanner, (b) ASAS, and (c) Digital camera.

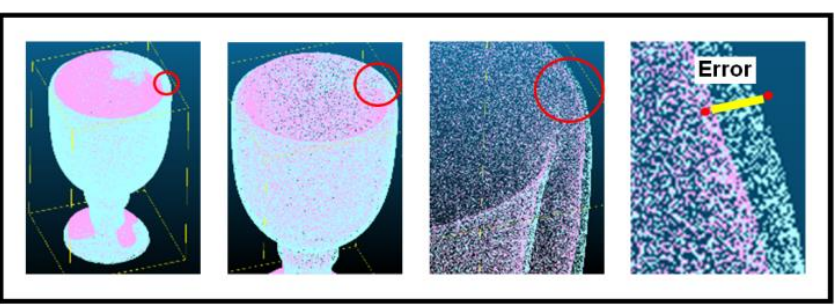

Fig. 9. The 'nearest neighbor distance' for each point of the compared cloud.

$$
\% \text { Error }=\frac{\text { Outlier Points }}{\text { Total Points }} \times 100
$$

where:

Outlier Points $=$ The distance of any compared points is greater than a $0.05-\mathrm{mm}$

Total Points $=$ The total points of the master model

\section{CONCLUSION}

In order to select the appropriate optical acquisition technique to create a new design quickly, presented in Table II are the recommendations obtained from the experiments of 
three different optical methods: 3D laser scanner, ASAS, and image-based acquisition.

For an object that contains various features on some portions of the surface, 3D laser scanner is required. However, the surface preparation is required before scanning attempt for getting a complete result.

ASAS presents as the appropriate technique for acquiring an object that contains small size and curvature shape, and the references are not required. For supporting direct interfacing between reverse engineering (RE) and rapid prototyping (RP) where the organized contour points can be sent directly to fabricate a prototype layer by layer, ASAS technique is recommend. However, the topmost and the bottommost areas cannot be detected. Using another two techniques are more effective.

When less acquisition time, low cost of equipment while preserving colors of the product are required, a digital camera is recommended. Using this technique can help the designer to extract and translate the hidden needs of the customers through images instead of spending very long and confused conversations.

TABLE II: COMPARISON RESULTS OF STUDIED TECHNOLOGIES

\begin{tabular}{llccc}
\hline & & Laser & ASAS & Camera \\
& & Scanner & & \\
\hline 1. Percentage of error & $15.27 \%$ & $8.42 \%$ & $16.28 \%$ \\
2. Equipment Cost & High & Medium & Low \\
\hline 3. Reference Point & Yes & No & Yes \\
4. Preparing Time & $15 \mathrm{~min}$ & $15 \mathrm{~min}$ & $5 \mathrm{~min}$ \\
\hline 5. Acquiring Time & $35 \mathrm{~min}$ & $18 \mathrm{~min}$ & $4 \mathrm{~min}$ \\
\hline 6. Reconstruction Time & $1 \mathrm{~min}$ & $5 \mathrm{~min}$ & $15 \mathrm{~min}$ \\
\hline 7. Surface Fitting Time & $10 \mathrm{~min}$ & $10 \mathrm{~min}$ & $15 \mathrm{~min}$ \\
\hline 8. Technical Skill & Medium & High & Low \\
\hline
\end{tabular}

\section{REFERENCES}

[1] S. Xiang, S. Chen et al., "Study on fast linear scanning for a new laser scanner," Optics \& Laser Technology, vol. 4, pp. 42-46, February 2010.

[2] A. M. Zoubir, "Introduction to statistical signal processing," in Academic, A. M. Zoubir, M. Viberg, R. Chellappa, and S. Theodoridis, Eds. Elsevier: Library in Signal Processing Press, 2014, ch. 1, pp. 3-7.

[3] C. Valentina et al., Journal of Biomechanics, vol. 49, vol. 3, pp. 127-131, 2016

[4] W. Yutao and H. Feng, "Effects of scanning orientation on outlier formation in 3D laser scanning of reflective surfaces," Optics and Lasers in Engineering, vol. 81, pp. 35-45, 2016.

[5] A. Wuttimanop and S. Rianmora, Manufacturing Systems Engineering, pp. 456-462, 2013.

[6] K. Lymer, "Image processing and visualisation of rock art laser scans from Loups's Hill," Digital Applications in Archaeology and Cultural Heritage, vol. 2, pp. 155-165, 2015.

[7] C. Yang, "Finest image sharpening by use of the modified mask filter dealing with highest spatial frequencies," International Journal for Light and Electron Optics, vol. 125, pp. 1942-1944, 2014.

[8] R. Yong et al., "Image retrieval: Current techniques, promising directions, and open issues," Communication and Image Representation, vol. 10, pp. 39-62, 1999.
[9] R. M. Haralick, and G. L. Kelly, "Pattern recognition with measurement space and spatial clustering for multiple images," Proceedings of the IEEE, vol. 57, pp. 654-665, April 1969.

[10] Y. Hu and L. Nie, "An aerial image recognition framework using discrimination and redundancy quality measure," Communication and Image Representation, vol. 37, pp. 53-62, May 2016.

[11] D. Needham, "Reverse engineering of the low temperature-sensitive liposome (LTSL) for treating cancer," Biomaterials for Cancer Therapeutics, pp. 270-348, 2013.

[12] A customer oriented methodology for reverse engineering software selection in the computer aided inspection scenario, Computers in Industry, vol. 67, pp. 54-71, 2015.

[13] C. Gopinath and G. Prasad, "Optimum material evaluation for gas turbine blade using reverse engineering (RE) and FEA," Procedia Engineering, vol. 97, pp. 1332-1340, 2014.

[14] T. Lee et al., "Reverse engineering a CCTV system, a case study," Digital Investigation, vol. 11, pp. 179-186, 2014.

[15] E. Anders et al., "Medical image processing on the GPU - Past, present and future," Medical Image Analysis, vol. 17, pp. 1073-1094, 2013.

[16] M. Christian et al., "3D bioprinting for engineering complex tissues," Journal of Biotechnology Advances.

[17] G. Niyati et al., "3D Gesture-recognition based animation game procedia,"Computer Science, vol. 45, pp. 712-717, 2015.

[18] R. Fernando et al., "3D animation of the transpyrenean corridor created by GIS tools," Procedia-Social and Behavioral Sciences, vol. 160, pp. 244-253, 2014.

[19] B. Banu, "The graphic design and application problems on the theatre publicity stands: A three-dimensional (3D) advertiser graphic design for a play," Procedia-Social and Behavioral Sciences, vol. 15, pp. 658-661, 2011.

[20] L. Ghang et al., "Usability principles and best practices for the user interface design of complex 3D architectural design and engineering tools," Human-Computer Studies, vol. 68, pp. 90-104, 2010.

[21] Development of a method for visualization and evaluation of production logistics in a multi-variant production, Procedia CIRP, vol. 17, pp. 481-486, 2014.

[22] Predicting sensorial attribute scores of ornamental plants assessed in $3 \mathrm{D}$ through rotation on video by image analysis: A study on the morphology of virtual rose bushes, Computers and Electronics in Agriculture, vol. 121, pp. 331-346, 2016.

[23] M. Aristeidou and N. Spyropoulou, "Building technology and science experiences in 3D virtual world," Procedia Computer Science, vol. 65, pp. 259-268, 2015.

[24] R. Trubka et al., "A web-based 3D visualisation and assessment system for urban precinct scenario modelling," ISPRS Journal of Photogrammetry and Remote Sensing.

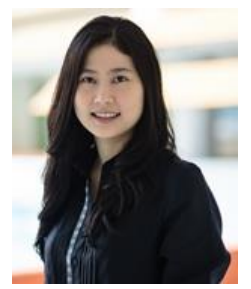

Suchada R. is an Assistant Professor in School of Manufacturing Systems and Mechanical Engineering, Sirindhorn International Institute of Technology, Thammasat University, Thailand. She received her D.Eng from Asian Institute of Technology, Thailand. Her research interests are reverse engineering, image-based acquisitions, and rapid prototyping.

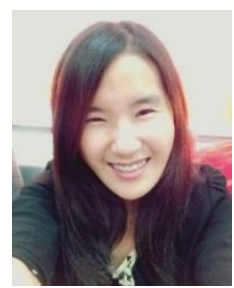

Molticha R. is a master student in Logistics and Supply Chain Systems Engineering Program, School of Manufacturing Systems and Mechanical Engineering, Sirindhorn International Institute of Technology, Thammasat University, Thailand. She received a B.Eng in Industrial Engineering, from Sirindhorn International Institute of Technology, Thammasat University, Thailand in 2013. 